PUPT 1576, IASSNS 95/92

hep-th/9511064

November 1995

\title{
Internal Structure of Black Holes
}

\author{
FinN LARSEN * \\ Department of Physics \\ Joseph Henry Laboratories \\ Princeton University \\ Princeton, N.J. 08544
}

FRANK WILCZEK ${ }^{\dagger}$

School of Natural Sciences

Institute for Advanced Study

Olden Lane

Princeton, N.J. 08540

\footnotetext{
* Research supported in part by a Danish National Science Foundation Fellowship. larsen@puhep1.princeton.edu

$\dagger$ Research supported in part by DOE grant DE-FG02-90ER40542. wilczek@sns.ias.edu
} 


\begin{abstract}
We present a number of qualitative arguments which strongly suggest that extremal dyonic black holes in the 4-dimensional low energy, classical field theory limit of toroidally compactified heterotic string theory represent largely degenerate classes of states in the quantum theory. We propose a simple expression for the full non-perturbative degeneracy, which contains no free continuous parameters and reproduces the Bekenstein-Hawking $S=\frac{A}{4 G_{N}}$ in the large-area limit (with the $\frac{1}{4}$ arising from microphysics). We sketch the elements of a physical picture leading to this expression: the holes support much hair, such that in counting it we are led to an effective string theory, and a matching condition whose solutions give the degeneracy.
\end{abstract}




\section{Introduction}

The thermodynamic entropy of a black hole is given by the BekensteinHawking formula

$$
S_{\text {therm }}=\frac{A}{4 \hbar G_{N}},
$$

where $A$ is the area of the black hole [1-2]. For zero-temperature holes, it is natural to attempt to identify this entropy with the one we expect from the fundamental principles of statistical mechanics:

$$
S_{\text {stat }}=\frac{1}{\hbar} \ln N
$$

where $N$ is the number of microscopically different states that the black hole can be in. Unfortunately the no-hair theorem - as commonly regarded - states that the conserved charges, measurable by observers far away, uniquely specify the black hole classically, and therefore the statistical mechanical entropy vanishes classically [3]. Quantum mechanics may change this, but it seems difficult to avoid that quantum effects only affect the $\mathcal{O}(1)$ corrections to the formulae above which, being of $\mathcal{O}\left(\frac{1}{\hbar}\right)$, must have a classical origin *.

Recently it has been suggested that string theory might improve this situation [4]. In important work Sen [5] offered a concrete mechanism in the context of a special class of extremal black holes in toroidally compactified heterotic string theory. He noted that keeping all conserved charges fixed there remain additional degeneracies among string states with the quantum numbers of the black hole, associated with freedom in the choice of transverse oscillation. The holes considered by Sen have zero area, and therefore zero entropy, classically, so they offer a limited perspective on the problem mentioned above. Cvetic and various collaborators [67] have generalized the solutions used by Sen to a class including extremal holes with non-zero area, and these provide an appropriate arena to consider the general

\footnotetext{
$\star$ In what follows, we will set $\hbar=1$.
} 
problem. They retain important technical features of the special case: in particular, they are BPS saturated states with $N=1$ (but not $N=2$ ) supersymmetry [8].

Here we consider the structure of these black holes, and attempt to identify the required degrees of freedom. The essence of the matter is that the no-hair "theorem" is not a law of Nature - indeed, isolated exceptions have been noted on several occasions [9]. In the field theories that arise as the low-energy limit of string theory, one finds that they fail in a spectacular way: appropriate black holes exhibit a string theory's worth of degeneracy, with a string tension renormalized from the microscopic value in a way that depends on the properties of the hole. This classical hair provides a microphysical grounding for the Bekenstein-Hawking entropy.

\section{Classical Black Holes and Suggestions of a State-Count}

We now briefly review the properties of the black holes of interest, and take special note of some very special properties of the area, which already suggest a state-counting interpretation.

We consider the low energy limit of heterotic string theory compactified on a generic torus. In Einstein metric the effective 4-dimensional action is

$$
\begin{aligned}
L=\frac{1}{16 \pi G_{N}} & \int d^{4} x \sqrt{-g}\left[R_{g}-\frac{1}{2} \partial^{\mu} \phi \partial_{\mu} \phi-\frac{1}{12} e^{-2 \phi} H_{\mu \nu \rho} H^{\mu \nu \rho}\right. \\
& \left.-G_{N} e^{-\phi} F_{\mu \nu}^{(a)}(L M L)_{a b} F^{(b) \mu \nu}+\frac{1}{8} \operatorname{Tr}\left(\partial^{\mu} M L \partial_{\mu} M L\right)+\text { fermion terms }\right]
\end{aligned}
$$

The fermion terms are determined by supersymmetry from the ones given. $H^{\mu \nu \rho}=$ $-\frac{1}{\sqrt{-g}} e^{2 \phi} \epsilon^{\mu \nu \rho \sigma} \partial_{\sigma} \Psi$ defines the axion $\Psi$ which we will be assume to vanish, with no essential loss of generality. The vacuum expectation value of the dilaton $\phi$ is related to the string coupling through $\left\langle e^{\phi}\right\rangle=e^{\phi_{\infty}}=g_{\mathrm{st}}^{2}$. The dimensionful string parameter $\alpha^{\prime}$ has been scaled out using $G_{N}=\frac{\alpha^{\prime} g_{\text {st }}^{2}}{4} . F_{\mu \nu}^{(a)}$ represents $28 U(1)$-gauge fields. The matrix $M$ parameterizes the metric of the compactified dimensions and 
other background fields. It satisfies

$$
M L M^{T}=L, \quad M^{T}=M, \quad L=\left(\begin{array}{ccc}
0 & I_{6} & 0 \\
I_{6} & 0 & 0 \\
0 & 0 & -I_{16}
\end{array}\right)
$$

Now consider extremal black hole solutions of this classical field theory. The asymptotic behavior of $F_{\mu \nu}^{(a)}$ at infinity identifies 28 electric charges $\vec{Q}$ and 28 magnetic charges $\vec{P}$. For the supersymmetric black holes that we are interested in, the full solution depends only on these charges. Specifically, the dependence on $g_{\mathrm{st}}$ and the background $M_{\infty}$ is determined by symmetries of the Lagrangean. Moreover, taking $\Omega \in \mathcal{O}(6,22)$ (i.e. $\Omega^{T} L \Omega=L$ ), the action is invariant under the T-duality transformation $M \rightarrow \Omega M \Omega^{T}$ and $A_{\mu}^{(a)} \rightarrow \Omega_{a b} A_{\mu}^{(b)}$ with the other fields kept fixed. Therefore the solution depends only on T-invariant combinations of the charges. For vanishing axion field it can be shown [6] that supersymmetry imposes the constraints $\vec{Q}^{T}\left[(L M L)_{\infty} \pm L\right] \vec{P}=0$. The remaining T-invariants are

$$
Q_{R, L}=\left|\vec{Q}^{T}\left[(L M L)_{\infty} \pm L\right] \vec{Q}\right|^{\frac{1}{2}}
$$

and similarly for $P$. The extremal black hole solutions are explicitly known [6] in terms of the T-invariants but we will only need the relations

$$
\begin{aligned}
G_{N} M_{\mathrm{ADM}}^{2} & =\frac{1}{2}\left[\frac{1}{g_{\mathrm{st}}} P_{R}+g_{\mathrm{st}}\left(\frac{Q_{R}}{g_{\mathrm{st}}^{2}}\right)\right]^{2} \\
S_{\text {therm }} & \equiv \frac{A}{4 G_{N}}=2 \pi \sqrt{\frac{\left(P_{R}^{2}-P_{L}^{2}\right)\left(Q_{R}^{2}-Q_{L}^{2}\right)}{g_{\mathrm{st}}^{4}}}
\end{aligned}
$$

Regular solutions satisfy $P_{R} \geq P_{L}$ and $Q_{R} \geq Q_{L}$.

Due to the normalization of the kinetic energy of the gauge field it is $e^{-\phi} F_{\mu \nu}^{(a)}$ rather than $F_{\mu \nu}^{(a)}$ that leads to a conserved charge. The Bianchi identity, on the other hand, is the standard one so the conserved charges are $\frac{\vec{Q}}{g_{\mathrm{st}}^{2}}$ and $\vec{P}$. The mass relation has been extensively studied recently [10-11]. Keeping $G_{N}$ and the 
conserved charges fixed, the black holes with vanishing magnetic charge are light for small coupling $g_{\mathrm{st}}$, and we expect them to be described by elementary string states. In contrast, all magnetically charged black holes are heavy for small coupling, and we expect them to be described by non-perturbative, or collective, states.

The entropy as defined above is simply a geometric parameter of the classical solution. Yet it exhibits several remarkable features, which are not at all implicit in its definition, and which strongly suggest a state-counting interpretation. Specifically, unlike $P_{R}$ and $P_{L}$ separately the combination $P_{R}^{2}-P_{L}^{2}$ is independent of the moduli $M_{\infty}$. The same is true for $Q$, and hence for the "entropy" $S_{\text {therm }}$. This is reminiscent of the classical independence of the phase space volume under canonical transformations - or, more directly in our context, the expected independence of the number of states with given conserved quantum numbers at infinity on adiabatic changes of underlying parameters. Also, when expressed in terms of conserved charges the entropy is independent of the string coupling. Again, this is consistent with one's expectation that parameters of the states (such as the mass), but not their total number, may depend on the coupling constant. The independence of coupling constant, more rigorously grounded in the principle of BPS saturation, is also welcome on technical grounds: it can be convenient to count the states for an arbitrarily weakly coupled theory.

\section{Heuristic Counting of String States}

In classical Einstein-Maxwell theory an extremal black hole configuration is described uniquely in terms of the electric and magnetic charges. In string theory such a state may have some degeneracy $d(\vec{Q}, \vec{P})$. This quantity is meaningful even in the interacting theory where the internal structure of the states may be complicated. In this section we will determine the degeneracy using general principles, and a natural hypothesis.

First, string theory respects T-duality, so a priori the degeneracy may depend on the T-invariants $P_{R, L}$ and $Q_{R, L}$ as well as the string coupling $g_{\mathrm{st}}$. However, 
the dependence on continuous parameters $M_{\infty}$ (implicit in $P_{R, L}$ and $Q_{R, L}$ ) and $g_{\mathrm{st}}$ must respect that the degeneracy is an integer. If we assume that the degeneracy is an analytic function of its variables, the most we can reasonably hope is that it takes on integer values at discrete points. Thus plausibly the only independent parameters that can appear as arguments of $d$ are the quantized charges $\frac{P_{R}^{2}-P_{L}^{2}}{2}$ and $\frac{Q_{R}^{2}-Q_{L}^{2}}{2 g_{\mathrm{st}}^{4}}$. (Our normalization is such that these two variables take on all integer values.) Next, let us take into account the symmetry under S-duality, which is conjectured to be an exact quantum symmetry of the full non-perturbative string theory [11]. In the absence of an axion, S-duality interchanges $\vec{P} \leftrightarrow \frac{1}{g_{\mathrm{st}}^{2}} \vec{Q}$ and $g_{\mathrm{st}} \leftrightarrow \frac{1}{g_{\mathrm{st}}}$. Thus the most general expression consistent with symmetries takes the form

$$
d(\vec{Q}, \vec{P})=F\left(\frac{Q_{R}^{2}-Q_{L}^{2}}{2 g_{\mathrm{st}}^{4}}+\frac{P_{R}^{2}-P_{L}^{2}}{2}, \frac{\left(Q_{R}^{2}-Q_{L}^{2}\right)\left(P_{R}^{2}-P_{L}^{2}\right)}{4 g_{\mathrm{st}}^{4}}\right)
$$

For black holes with vanishing magnetic charge we can calculate the degeneracy in perturbation theory, following Sen [5]: the mass of a string state is given in terms of its charges by

$$
G_{N} M_{\mathrm{ADM}}^{2}=g_{\mathrm{st}}^{2}\left(\frac{1}{2 g_{\mathrm{st}}^{4}} Q_{R}^{2}+N_{R}-\frac{1}{2}\right)=g_{\mathrm{st}}^{2}\left(\frac{1}{2 g_{\mathrm{st}}^{4}} Q_{L}^{2}+N_{L}-1\right)
$$

For extremal states the supersymmetry algebra determines $N_{R}=\frac{1}{2}$, so we recover the mass formula from the black holes in the previous section. This is the identification between black holes and string states found in [10]. The second equality is the level matching condition, which enforces reparameterization invariance along the string. For a given set of charges the oscillator level $N_{L}$ is given by (3.2) but there any many ways that oscillations can be distributed on individual string modes. The degeneracy is

$$
d(\vec{Q}, \vec{P}=0) \equiv f\left(N_{L}\right) \simeq e^{4 \pi \sqrt{N_{L}}}
$$

The function $f\left(N_{L}\right)$ is known, but complicated, so we use its leading term for large 
$N_{L}$. The statistical entropy is

$$
S_{\text {stat }}=\ln f\left(N_{L}\right) \simeq 4 \pi \sqrt{\frac{Q_{R}^{2}-Q_{L}^{2}}{2 g_{\mathrm{st}}^{4}}+1}
$$

For vanishing magnetic charge the corresponding thermodynamic entropy calculated in the previous section is zero. However, it has been argued that the thermodynamic entropy receives corrections of a form consistent with the statistical entropy above $[5,12]$.

The perturbative calculation determines the dependence of the general degeneracy on $\frac{Q_{R}^{2}-Q_{L}^{2}}{2 g_{\mathrm{st}}^{4}}+\frac{P_{R}^{2}-P_{L}^{2}}{2}$. Unfortunately the other variable $\frac{\left(Q_{R}^{2}-Q_{L}^{2}\right)\left(P_{R}^{2}-P_{L}^{2}\right)}{4 g_{\mathrm{st}}^{4}}$ vanishes for zero magnetic charge, so the perturbative calculation teaches us nothing about its significance. Specifically, the general relation might, a priori, be independent of this parameter. We shall now argue this cannot be true. Consider the regime where at least one of the charges is large. Consider the number of states consistent with asymptotic charges $\overrightarrow{P_{1}}+\overrightarrow{P_{2}}$ and $\overrightarrow{Q_{1}}+\overrightarrow{Q_{2}}$. A subclass of such states consists of well separated subsystems each carrying part of the charge. We therefore expect

$$
d\left(\overrightarrow{Q_{1}}+\overrightarrow{Q_{2}}, \overrightarrow{P_{1}}+\overrightarrow{P_{2}}\right) \geq d\left(\overrightarrow{Q_{1}}, \overrightarrow{P_{1}}\right) d\left(\overrightarrow{Q_{2}}, \overrightarrow{P_{2}}\right)
$$

It requires the degeneracies to be at least exponential in electric as well as magnetic charges, and is inconsistent with $F$ being independent of its second argument.

As an ansatz let us assume the existence of a generalized matching condition equating $N_{L}$ to some function of $\vec{Q}$ and $\vec{P}$. In view of the clustering property, this function can not simply be the sum of terms depending only on $\vec{Q}$ and $\vec{P}$ separately. As the next simplest choice let us consider a product of terms depending on $\vec{Q}$ and $\vec{P}$ respectively. In fact, taking into account the known result for $P=0$, there is a unique factorized matching condition

$$
N_{L}=\left(\frac{Q_{R}^{2}-Q_{L}^{2}}{2 g_{\mathrm{st}}^{4}}+1\right)\left(\frac{P_{R}^{2}-P_{L}^{2}}{2}+1\right),
$$

It is an independent consistency check, although admittedly weak, that this relation 
equates integers. Thus we are led to

$$
S_{\text {stat }}=\ln f\left(N_{L}\right) \simeq 4 \pi \sqrt{\left(\frac{Q_{R}^{2}-Q_{L}^{2}}{2 g_{\mathrm{st}}^{4}}+1\right)\left(\frac{1}{2}\left(P_{R}^{2}-P_{L}^{2}\right)+1\right)}
$$

Remarkably, this expression reproduces $S_{\text {therm }}$ for large charges. In particular, it gives the numerical coefficient in front of the area correctly. In its entirety it is a corrected form of the thermodynamic entropy formula, that interpolates between Sen's perturbative result and the classical expression.

In the following section we shall consider the problem from a more microscopic perspective, and argue that the existence of a matching condition in factorized form is not only esthetically pleasing but also physically necessary. Taken together with the earlier arguments, this singles out (3.7) uniquely. Let us note the microphysical origin of the various coefficients. The 1's that quantum correct the electric and magnetic charges arise from world sheet zero point energy. The coefficient in front

of the square root is actually $2 \sqrt{\frac{\pi^{2}}{6} \times 24}$ which is related to the world sheet entropy $\frac{\pi^{2}}{6}$ of the 24 transverse oscillators of the left movers of the heterotic string.

\section{Black Hole Hair}

The static black hole solution is specified uniquely by $\vec{Q}, \vec{P}$, and the extremeality condition [6]. In a generic theory one would expect that time-dependent perturbations are exponentially damped outside the horizon - black holes have no hair [3]. However string theory dictates additional internal dimensions and a very special matter content so, perhaps surprisingly, the black hole allows carefully chosen perturbations. To exhibit these in a simple form we use the string metric $G_{\mu \nu}=e^{\phi} g_{\mu \nu}$. We also single out one of the compactified directions, $x^{9}$ say, and let the two independent electric charges of the background be associated with the Kaluza-Klein field $G_{9 t}$ and the antisymmetric field $B_{9 t}$, with the other internal 
dimensions carrying no electric charge. The modes

$$
\begin{aligned}
G_{u i}(u, r) & =\partial_{u} X^{i}(u) C(r)=B_{u i}(u, r) ; \quad i=1, \ldots, 8 \\
A_{u}^{J}(u, r) & =\partial_{u} X^{J+8}(u) C(r) ; \quad J=1, \ldots, 16
\end{aligned}
$$

where $u=x^{9}-t$ and $v=x^{9}+t$, propagate freely in this background. The functions $X^{I}(u)(I=1, \ldots, 24)$ are arbitrary, except that they must respect the periodicity of the compactified coordinate $x^{9}$. The profile function is $C(r)=\frac{g_{\mathrm{st}}^{2}}{r+\sqrt{\alpha^{\prime} / 2}\left(Q_{R}-Q_{L}\right)}$. Using the techniques of $[7,13-14]$, each of the perturbations can be extended to exact solutions, respecting supersymmetry. This even holds at the level of the exact conformal field theory, which is the string-theoretic analogue of a classical state . There is a simple physical interpretation of this hair: the translational invariance of the underlying 10-dimensional theory is broken by the location of the black hole. After gauge-fixing this leads to $8 \mathrm{Nambu-Goldstone} \mathrm{modes} \partial_{u} X^{i}$. Analogously the internal 16-torus leads to the remaining $\partial_{u} X^{I}$. As we shall explain below, the background allows only left moving perturbations, and no dependence on internal coordinates other than $x^{9}$.

In deriving equations of motion from the action we are instructed to consider variations of the fields, keeping them fixed on the boundaries. Besides the bulk Euler-Lagrange equations, this implies regularity conditions at real or coordinate singularities. In the present context, by considering the coefficient in variations of $\partial_{r} G_{v v}$ near the horizon $r=0$ we are led - after a substantial calculation - to a matching condition for the left movers

$$
\sum_{I=1}^{24}\left[\partial_{u} X^{I}(u)\right]^{2}=\frac{1}{g_{\mathrm{st}}^{4}}\left(Q_{R}^{2}-Q_{L}^{2}\right)
$$

Right movers are prohibited by the similar argument, with $u$ replaced by $v$. Since the electric charge is in the $x^{9}$ direction, the corresponding condition in directions $\bar{u}=x^{i}-t$ (with $\left.i=4, \ldots, 8\right)$ forbids oscillation. This is why we only find a string's worth of oscillators, rather than those characteristic of some higher p-brane. These 
matching conditions should be considered as a mathematical embodiment of the requirement that there are no physical sources at the horizon. For electric black holes Callan et. al. [13] obtain a generalized matching condition of the same form by demanding a form of cosmic censorship. Closely related matching conditions occur in other contexts [15].

The low energy dynamics of black holes is governed by that of the 0-modes, which in turn realize an effective heterotic string theory with the right movers in their ground states. To count states we must find the normalization of the functions $X^{I}(u)$; more precisely, the number of states in the semi-classical approximation is determined by the volume of classical phase space. The momentum conjugate to the collective coordinate $X^{I}$, denoted $\Pi^{I}$, is given in light-cone quantization (with $v$ as the "time") by the variational derivative of the Lagrangean with respect to $\partial_{v} X^{I}$. Note that while $\partial_{v} X^{I}=0$ on any solution of the equation of motion, the momenta need not vanish. The result must be of the form $\Pi^{I}=\frac{T}{2} \partial_{u} X^{I}$, which after quantization leads to the generalized matching condition

$$
N_{L}=\left(\pi \alpha^{\prime} T\right) \frac{Q_{R}^{2}-Q_{L}^{2}}{g_{\mathrm{st}}^{4}}
$$

up to a normal ordering constant. $N_{L}$ is the oscillator level of the 240 -modes.

Due to a scaling symmetry of the classical action, the purely electrical black hole depends only on the parameters $\sqrt{\alpha^{\prime}} \frac{Q_{R, L}}{r}$. In the weak coupling limit, keeping the conserved charges $\frac{Q_{R, L}}{g_{\mathrm{st}}^{2}}$ fixed, the black hole reduces to flat space. In this limit, assuming that states of the effective string theory are to be identified with elementary string states with the same quantum numbers, we must put $T=\frac{1}{2 \pi \alpha^{\prime}}$. It is a non-trivial coincidence, that the properly normalized classical regularity condition for 0 -modes in an electric background agrees with the elementary string matching condition.

Adding magnetic charges, the black hole background depends on $\sqrt{\alpha^{\prime}} \frac{P_{R, L}}{r}$. In the weak coupling limit the tension depends on the magnetic, but not the electric 
charges. This is the origin of the factorization of the matching condition. Given this factorization, the general considerations of the previous section determines the degeneracy $d(\vec{Q}, \vec{P})$ uniquely, in the form previously mentioned.

Given the explicit form of the 0-modes the tension, determined indirectly above, can in principle be calculated directly. Since the required Hamiltonian formalism is both complex and notoriously subtle due to the need for careful attention to boundary terms, the following remarks should be regarded as indicative rather than definitive. We find for the bulk tension

$$
T=\frac{1}{2 \pi \alpha^{\prime}} \int d^{4} x \sqrt{-G} e^{-\Phi}\left[G^{u v} G^{i j} \partial_{i} C \partial_{j} C\right]
$$

where the integral is over three spatial directions and the internal dimension carrying the magnetic charges. In the purely electric case the tension is finite but vanishes as $g_{\mathrm{st}} \rightarrow 0$, so we fail to recover the flat space result. This is not unexpected from a physical point of view, because the action from which we derived the explicit form of the solution is not uniformly accurate. At scales of order $\mathcal{O}\left(\sqrt{\alpha^{\prime}}\right)$ close to the horizon we cannot trust the form of the metric to be inserted into (4.4). Fortunately the weak coupling limit determines the true tension, as we have seen.

In the generic case, one may take the electric charge smoothly to zero as the coupling becomes weak at infinity. The remaining magnetic soliton is controlled by an effective coupling which becomes of order unity at a large distance from the horizon. We assume - motivated by duality - that universal physics governs the interior strong coupling region. Concretely, we suppose the expression in brackets in (4.4) is universal and put $\sqrt{-G} e^{-\Phi} \simeq \frac{1}{2}\left(P_{R}^{2}-P_{L}^{2}\right)$ - its value where the coupling become strong. The matching condition then emerges in the anticipated, factorized, S-dual form.

While our technical implementation certainly leaves much room for improvement, the emergent physical picture seems compelling: the dyon has an aspect that is electric and singular at short distances, cut off in a universal manner; and also a 
soft solitonic, magnetic aspect which gives it a classical size, so that this universal physics is repeated over a large volume. Let us emphasize again that this qualitative picture, combined with the weak coupling limit, allows one to determine the degeneracy quantitatively.

\section{Discussion}

The idea that there is a very large density of states arising from the normalization of a degree of freedom associated with fluctuations of the horizon has also been proposed on apparently very different grounds, with no explicit reference to string theory but crucially involving a (notional) general covariant cut-off in quantum gravity, by Teitelboim [16]. We suspect there are deep connections between these points of view.

The black holes considered above are not as exotic as they might appear at first sight. For example, they include as a special case the extremal Reissner-Nordstrøm black holes [10]. Those correspond to $Q_{L}=P_{L}=0$ and $Q_{R}=P_{R}=\frac{g_{\mathrm{st}}}{\sqrt{2}} Q_{\mathrm{el}}$. This identification is possible because the dilaton decouples in this limit. The additional structure in our construction serves to embed standard electromagnetism in a theory that is supersymmetric, to avoid quantum corrections. It also allows a precise connection with string theory. Based on the adiabatic invariance of state number one would expect that, to the extent that all string theories are continuously connected, details of the embedding, the class of string theory, and the compactification are unimportant. We have seen this to be the case, for a restricted but non-trivial class of moduli. Because our picture of black hole internal structure classical hair - is so simple and concrete, we also expect that it applies to all black holes, extremal or not.

\section{acknowledgments}

We would like to thank C. Callan, D. Gross, J. Maldacena, and H. Verlinde for discussions. We have also benefited from suggestions by M. Cvetic, A. Peet, E. Sharpe, and L. Susskind. 


\section{REFERENCES}

1. J. Bekenstein, Lett. Nuov. Cimento 4 (1972) 737, Phys. Rev. D7 (1973) 2333, Phys. Rev. D9 (1974) 3292.

2. S. Hawking, Nature (Physical Science) 248 (1974) 30, Comm. Math. Phys. $43(1975)$.

3. R. Price, Phys. Rev. D5 (1972).

4. L. Susskind, Some Speculations about Black Hole Entropy in String Theory, hep-th/9309145.

5. A. Sen, Extremal Black Holes and Elementary String States, hep-th/9504147

6. M. Cvetic and D. Youm, Dyonic BPS Saturated Black Holes of Heterotic String on a Six Torus, hep-th/9507090.

7. M. Cvetic and A. Tseytlin, General class of BPS saturated dyonic black holes as exact superstring solutions, hep-th/9510097.

8. R. Kallosh, A. Linde, T. Ortin, A. Peet, and A.V.Proeyen, Supersymmetry as a cosmic censor, Phys. Rev. D46 (1992) 5278.

9. S. Coleman, J. Preskill, and F. Wilczek, Growing Hair on Black Holes, Phys. Rev. Lett. 67 (1991) 1975; and references therein.

10. M. J. Duff and J. Rahmfeld, Massive String States as Extreme Black Holes, Phys. Lett. B345 (1995) 441.

11. A. Sen, Strong-Weak Coupling Duality in Four Dimensional String Theory, Int. J. Mod. Phys. A9 (1994) 3702; C. M. Hull and P. K. Townsend, Unity of Superstring Dualities, Nucl. Phys. B438 109 (1995); E. Witten, String Theory in Various Dimensions, Nucl. Phys. B443 (1995) 85.

12. A. Peet, Entropy and Supersymmetry of D Dimensional Extremal Electric Black Holes versus String States, hep-th/9506200.

13. C. Callan, J. Maldacena, and A. Peet, Extremal Black Holes As Fundamental Strings, hep-th/9510134. 
14. G. T. Horowitz and A. A. Tseytlin, New class of exact solutions in string theory, Phys. Rev. D51 2896 (1995).

15. A. Dabholkar and J. Harvey, Phys. Rev. Lett. 63 (1989) 478; A. Dabholkar, G. Gibbons, J. Harvey, and F. Ruiz-Ruiz, Nucl. Phys. B340 (1990) 33.

16. C. Teitelboim, Action and Entropy of Extreme and Nonextreme Black Holes, Phys. Rev. D51 (1995) 4315, Statistical Thermodynamics of a Black Hole In terms of Surface Fields, hep-th/9510180 . 